\title{
Amino Acid Analysis Table
}

\begin{tabular}{|c|c|c|c|c|}
\hline $\begin{array}{c}\text { Amino } \\
\text { acid }\end{array}$ & $\begin{array}{l}\text { Experimental } \\
\text { for } s 4 a^{a}\end{array}$ & $\begin{array}{l}\text { Predicted } \\
{\text { for } s 4 a^{b}}^{b}\end{array}$ & $\begin{array}{l}\text { Experimental } \\
\text { for } s 4 b^{a}\end{array}$ & $\begin{array}{l}\text { Predicted } \\
\text { for } \mathbf{s} 4 b^{b}\end{array}$ \\
\hline$A s x^{c}$ & $2.07(2)$ & 2 & $2.23(2)$ & 2 \\
\hline$G \mid x^{c}$ & $0.98(1)$ & 1 & $2.11(2)$ & 2 \\
\hline Ser & $2.53(3)$ & 3 & $1.71(2)$ & 2 \\
\hline His & $0.00(0)$ & 0 & $0.00(0)$ & 0 \\
\hline Gly & $1.97(2)$ & 2 & $2.35(2)$ & 2 \\
\hline Thr & $3.71(4)$ & 4 & $4.82(5)$ & 5 \\
\hline Ala & $0.00(0)$ & 0 & $0.00(0)$ & 0 \\
\hline Arg & $1.00(1)$ & 1 & $1.00(1)$ & 1 \\
\hline Tyr & $0.84(1)$ & 1 & $0.99(1)$ & 1 \\
\hline Val & $1.73(2)$ & 2 & $2.03(2)$ & 2 \\
\hline Met & $0.90(1)$ & 1 & $0.97(1)$ & 1 \\
\hline Trp & $0.00(0)$ & 0 & $0.00(0)$ & 0 \\
\hline Phe & $0.00(0)$ & 0 & $0.00(0)$ & 0 \\
\hline Ile & $0.75(1)$ & 1 & $0.93(1)$ & 1 \\
\hline Leu & $1.03(1)$ & 1 & $1.14(1)$ & 1 \\
\hline Lys & 0.99 (1) & 1 & $4.33(4)$ & 4 \\
\hline Pro & $0.96(1)$ & $4^{\mathrm{d}}$ & $0.56(1)$ & $7^{d}$ \\
\hline Hypro & $3.24(3)^{\mathrm{e}}$ & - & $5.85(6)$ & - \\
\hline Cystine & $2.23(3)^{f}$ & $0^{f}$ & $2.17(3)^{f}$ & $0^{f}$ \\
\hline Cys & $0.00(0)^{f}$ & $6^{f}$ & $0.00(0)^{f}$ & $6^{f}$ \\
\hline
\end{tabular}

\section{Table legend:}

a Experimental amino acid contents are expressed as the molar ratio relative to arginine. The numbers in parentheses are the number of residues predicted based on the molar ratio.

b Predicted number of amino acids from cDNA sequences.

c Asn is converted to Asp and Gln to Glu as a result of acid hydrolysis.

$\mathrm{d}$ Gene-predicted sequences cannot differentiate between proline and post-translationally modified hydroxyproline. 
e The number of hydroxyprolines for s4a were assayed in a separate amino acid analysis. The molar ratio is relative to lysine.

f One cystine residue is equivalent to two (disulfide-bonded) cysteine residues, corresponding to six cysteines per peptide. Since the cysteines are linked during hydrolysis, the molar ratio tends to be lower.

Amino Acid Analysis. Amino acid analysis was performed by the Texas A\&M University Protein Chemistry Laboratory, according to a reported procedure (1).

For s4a, analysis indicates three serine residues, in agreement with the cDNA predicted sequence. Additionally, four threonine residues $(T)$, two glycine $(\mathrm{G})$, and two valine $(\mathrm{V})$ are identified. A single residue each is indicated for arginine (R), tyrosine $(\mathrm{Y})$, methionine $(\mathrm{M})$, isoleucine (I), leucine (L), lysine (K), and proline (P). The cDNA-predicted sequence indicates the presence of three more proline residues. The missing proline is due to post-translational modification (PTM) of the proline to hydroxyproline. Although hydroxyproline was not assayed in this particular amino acid analysis, the presence of these three residues by mass spectrometric (MS) analysis of the intact and Asp-N-cleaved fragments strongly supports this conclusion. Further, tandem MS experiments verify both the presence and location of these modified residues. Due to the acid hydrolysis conditions, asparagine (N) is converted to aspartate (D); these residues cannot be distinguished from each other and are reported as Asx. Two Asx residues were identified in the s4a amino acid analysis, matching sequence predictions for one $\mathrm{D}$ and one N. Similarly, acid hydrolysis converts glutamine (Q) to glutamate (E); the resulting residue is labeled Glx. One Glx residue was noted in the analysis corresponding to the predicted N-terminal glutamine (Q) seen in the cDNA sequence. Each of the cysteine (C) residues in the predicted sequence is likely disulfide bonded, as is the case for all conotoxins. Amino acid analysis cleaves $\mathrm{C}$ residues from adjacent amino acids but disulfide bonding remains unaffected by acid hydrolysis; thus, the $\mathrm{C}$ residues likely stay together in pairs and are reported as cystine. The three reported cystine residues correspond to six actual $\mathrm{C}$ residues, each of which is involved in disulfide bonding. This result is also representative of the disulfide-rich nature of cone snail toxins in general. As mentioned previously, the predicted sequence yields a glycine residue at the cleaved C-terminal that results in amidation of the cysteine residue and a mass difference of $-1 \mathrm{Da}$. The presence of $\mathrm{Q}$ at the $\mathrm{N}$-terminal is strongly suggestive of pyroglutamate (pGlu) as a PTM and a mass difference of $-17.01 \mathrm{Da}$. The average protonated mass of the residues determined for s4a, including disulfide bonding, hydroxylation of proline (2), and pGlu and Cterminal amidation was calculated to be $3191 \mathrm{Da}$.

The fraction corresponding to the second-most abundant peak, s4b, was also subjected to amino acid analysis. The results and corresponding cDNA matches are illustrated in the main text. Notable differences in the predicted sequences of s4b and s4a were then confirmed by amino acid assay. Specifically, the two cDNA-predicted serine residues, in contrast to three in s4a, were corroborated by the amino acid analysis results. Additionally, five $\mathrm{T}$ residues and four $\mathrm{K}$ residues were matched. Two residues each of $\mathrm{G}$ and $\mathrm{V}$, and single residues of $\mathrm{R}, \mathrm{Y}, \mathrm{M}, \mathrm{I}$, and $\mathrm{L}$ were detected, all matching the predicted sequence. The cDNA results indicate a total of seven proline residues. As mentioned previously, these amino acids can be post-translationally modified to form hydroxyproline, especially in cone snail toxins. For this analysis, an assay for 
hydroxyproline was performed and the results indicated that the remaining six residues from the predicted sequence were in fact hydroxyproline. The two reported Asx residues correspond to D and $\mathrm{N}$ in the predicted sequence. There were also two Glx residues indicated in the assay that match $\mathrm{Q}$ and $\mathrm{E}$ in the predicted sequence. As with s4a, the three reported cystine residues in the acid hydrolysis assay correspond to the remaining six disulfide-bonded $\mathrm{C}$ residues in the cDNApredicted sequence; this is further supported by the report of no Cys residues (nondisulfide bonded cysteine). Similar to the s4a predicted sequence, a glycine present at the C-terminal cleavage site indicates the presence of C-terminal amidation and a mass shift of $-1 \mathrm{Da}$. The presence of $\mathrm{Q}$ at the $\mathrm{N}$-terminal of $\mathrm{s} 4 \mathrm{~b}$ is likewise a marker for potential $\mathrm{N}$-terminal modification to pGlu. In the case of pyroglutamate formed from glutamine, the mass difference is $-17.01 \mathrm{Da}$. The average protonated mass of the residues determined for $\mathrm{s} 4 \mathrm{~b}$, including disulfide bonding and pGlu and C-terminal amidation, was calculated to be 4058 .

\section{References:}

(1) Godel, H., Seitz, P., and Verhoef, M. (1992) Automated amino acid analysis using combined OPA and FMOC-Cl precolumn derivatization, LCGC 5, 44-49.

(2) Craig, A. G., Bandyopadhyay, P., and Olivera, B. M. (1999) Post-translationally modified neuropeptides from Conus venoms, Eur. J. Biochem. 264, 271-275. 